\title{
Use of Accelerators for Biomechanical Analysis of Walking Motion Aided by Wheeled Walking Frames
}

\author{
Satoru Okamoto \\ Interdisciplinary Faculty of Science and Engineering, Shimane University, Matsue 690-8504, Japan
}

Received: July 01, 2014 / Accepted: July 25, 2014 / Published: September 25, 2014.

\begin{abstract}
Walkers improve self-reliability. We examined the effectiveness of a newly developed wheeled walking frame for use by physically handicapped persons. Unstable gaits in walker users were analyzed by tri-axial accelerometers and a motion capture system. Several markers were placed on subjects' backs and legs. Subjects were requested to walk around a test course at a comfortable speed, while their motion was recorded by two high-speed video cameras. The activities performed on the test course comprised standing, normal walking, fast walking, and walking over a barrier. Any accidental falls were also recorded. We established the characteristic rules of gait motion using a walker. Furthermore, we demonstrated that gait characteristics are more conveniently extracted from acceleration sensors than from motion capture systems, since the sensors can be affixed to subjects for self-monitoring and goal achievements. The methods employing acceleration sensors are considered suitable for determining the average gait motions of elderly persons living in nursing homes, and can be used to evaluate walking motion before and after rehabilitation.
\end{abstract}

Key words: Wheeled walking frame, gait motion, biomechanical analysis, acceleration sensor, high-speed video camera.

\section{Nomenclature}

$\begin{array}{ll}\text { FFT } & \text { Fast Fourier transform } \\ \text { RMS } & \begin{array}{l}\text { Root mean square } \\ T\end{array} \\ \text { TUG } & \begin{array}{l}\text { Time (s) } \\ \text { Timed "up and go" }\end{array} \\ X & \begin{array}{l}\text { Coordinate in the direction of walking motion } \\ \text { (forward-backward direction) }\end{array} \\ Y & \begin{array}{l}\text { Coordinate transverse to the walking motion } \\ \text { (right-left direction) }\end{array} \\ Z & \begin{array}{l}\text { Coordinate perpendicular to the floor surface } \\ \text { (vertical direction) } \\ \alpha\end{array} \\ \text { Acceleration }\left(\mathrm{m} \cdot \mathrm{s}^{-2} \text { ) }\right.\end{array}$

\section{Introduction}

Walking aids include canes (commonly referred to as walking sticks), crutches and walkers. These devices assist upright ambulation by improving stability, reducing lower limb loading, generating movement, or all of these [1]. Assistive devices for balance and mobility introduce benefits, demands and adverse consequences [2]. Walkers provide a more stable

Corresponding author: Satoru Okamoto, Ph.D., professor, research fields: fluid dynamics, energy system, visualization, biomechanics. E-mail: okamoto@cis.shimane-u.ac.jp. support base than other ambulation aids, but they are less versatile. They are effective on flat and level surfaces, but may present a problem on uneven surfaces or in narrow passages such as doorways. Walkers equipped with wheels may be rolled instead of being lifted. Most wheeled walkers are equipped with wheel locks or brakes [3, 4]. The kinematics and biomechanics of device-assisted walking [5], rollator walking [6] and abnormal walking have been widely investigated using a multibody model, which combines models of abnormal walking and walking aids to reduce compensatory action [7]. Other research has identified mediating factors that may determine the best prescription for a walking aid [8]. Change-in-support reactions for balance recovery, namely, control mechanisms, age-related changes and implications for fall prevention have also been investigated [9]. Kim et al. [10] reported the effect of rollator handle type on plantar foot pressure exerted by elderly women during walking. 
Acceleration sensors are effective tools for analyzing motion during exercise, and they have been adopted in several motion analysis studies. Using accelerometers alone, Takenoshita et al. [11] evaluated walking, hill climbing, standing and sitting motions, as well as obstacle avoidance. Sekine et al. [12] used a tri-axial acceleration sensor to compare the fractal dynamics of body motion in patients with Parkinson's disease and a physically unimpaired person.

However, few studies have utilized acceleration sensors to investigate walking motion [13]. The present study experimentally analyzed the walking motion of commercial walker users by attaching acceleration sensors to the subjects' lumbar region. The characteristics and size of the output signals were recorded for a range of motions, including standing, sitting, normal gait and turning gait. While the sensors acquired measurements, the video cameras recorded the subjects' images corresponding to the sensor signals. Therefore, the extent of rehabilitation in elderly subjects could be determined by comparing their motion before and after care, and the effectiveness of using a commercial walker was estimated solely from the sensor output signals. The study is limited by the number of subjects, and the subjects are not matched with the controls.

We then examined the effectiveness of a new wheeled walking frame developed for physically handicapped persons. The effectiveness of the wheeled walking frame was quantitatively determined from biomechanical walking motion analysis and the accelerations recorded by the acceleration sensor attached to the lumbar region. The quantitative and absolute criteria collectively enabled the evaluation of the walking motions. In a future study, acceleration sensors will be used to precisely determine the effect of the wheeled walking frame on walking motion, compared to walking without a commercial walker. The paper is organized as follows: Section 2 shows material and methods; Section 3 presents results; Section 4 presents discussions; and Section 5 gives conclusions.

\section{Material and Methods}

\subsection{Experimental Apparatus and Methods}

All motions including walking were filmed in three dimensions by two synchronized high-speed video cameras (NAC HSV-1000, NAC Image Technology Inc., Tokyo, Japan), using the direct linear transform method. Prior to the experiment, the cameras had been pre-connected by cables. Cameras A and B filmed from a diagonal and lateral direction, respectively, enabling the construction of three-dimensional images. The cameras were supplemented with adjacent lighting equipment and calibrated with a triangular pyramid calibration unit of length $1.00 \mathrm{~m}$ and height $1.45 \mathrm{~m}$. The control points were prevented from slipping by attaching them to the body with hooks and loop fasteners and wrapping joints in elbow and knee pads. A total of 20 control points were attached to the body and the four-wheel commercial walker (ETAC SL Four-Caster Walker from Sharp Training Co., Sweden, length $750 \mathrm{~mm}$, width from $560 \mathrm{~mm}$ to $400 \mathrm{~mm}$, height $800 \mathrm{~mm}$, wheel diameter $210 \mathrm{~mm}$ and weight 5.5 kg) (Fig. 1a). Physically handicapped subjects were allocated the newly developed wheeled walking frame (KW100 from KISHI ENGINEERING CO., length $890 \mathrm{~mm}$, width $600 \mathrm{~mm}$, height of seat $820 \mathrm{~mm}$, height of arm 1,160 mm, wheel diameter $310 \mathrm{~mm}, 150 \mathrm{~mm}$ and weight $23 \mathrm{~kg}$ ) (Fig. 1b).

The accelerations of the walking motion in the $X, Y$ and $Z$ directions were measured by tri-axial acceleration sensors (ONO SOKKI NP3560, ONO SOKKI, Tokyo, Japan; dimensions $10 \times 10 \times 10 \mathrm{~mm}^{3}$, weight $5 \mathrm{~g}$, maximum acceleration $4,900 \mathrm{~m} \cdot \mathrm{s}^{-2}$, impact resistance $98,000 \mathrm{~m} \cdot \mathrm{s}^{-2}$ ). These sensors were attached to the walker, lumbar region and right shin. They were attached to the body parts by processing jigs and fixed to prevent the slippage or detachment. Their purpose was to measure the vertical, horizontal and lateral motions. In Section 3.2, in the evaluation experiment, the acceleration sensors were placed immediately above the L3 spinous process which reflects the 
acceleration of the body's center of mass and fixed with a single elastic belt. A schematic of the experimental apparatus is shown in Fig. 2.

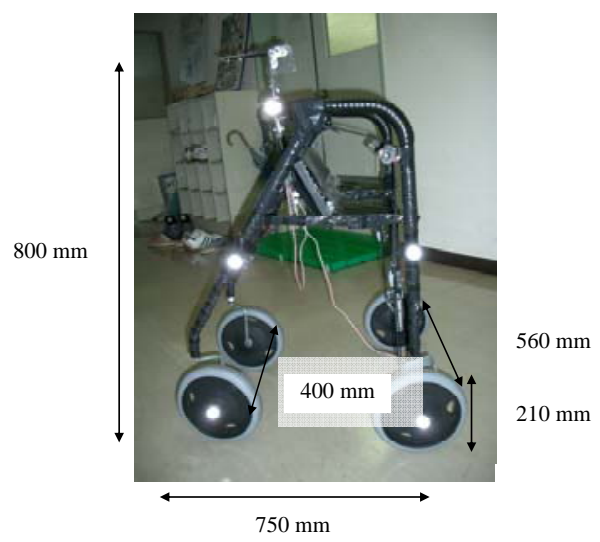

(a)

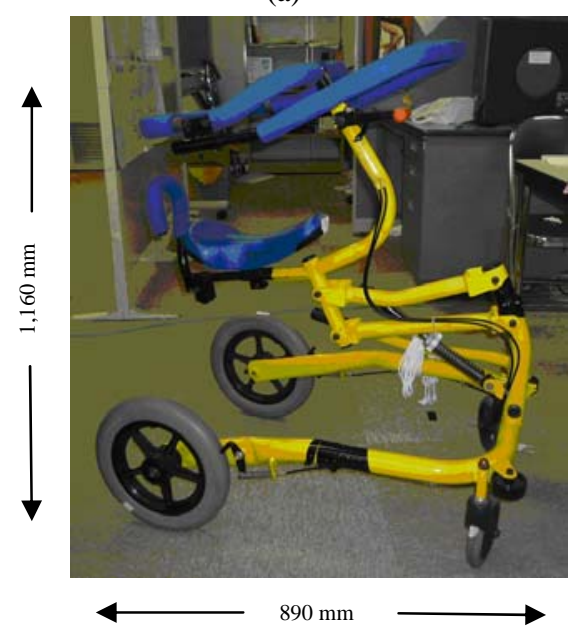

(b)

Fig. 1 (a) Commercial walker and (b) wheeled walking frame.

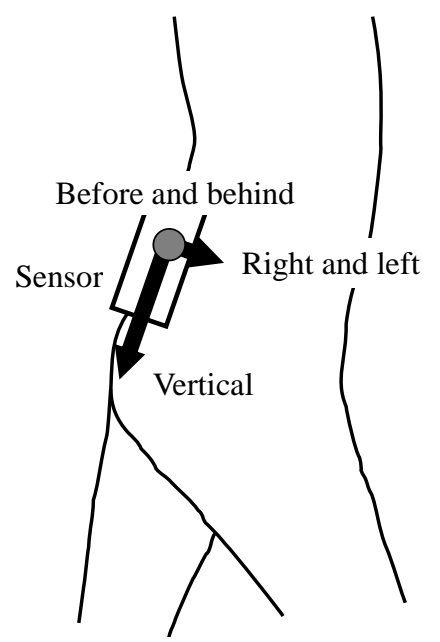

Fig. 2 Accelerations in three directions.

\subsection{Preliminary and Evaluation Experiments}

Two types of experiments were performed: (1) a preliminary experiment that recruited healthy university students and (2) an evaluation experiment involving designated elderly subjects using long-term health care facilities and patients using outpatient services. The commercial walker was used by all subjects in all measurements. The preliminary experiment acquired measurements during normal walking, motion over steps, standing, sitting and falling. In addition, standing and sitting activities were recorded while using and not using the brake on the walker. Falls caused by stumbling, and backward falls while sitting, were measured. A schematic of the experimental apparatus is shown in Fig. 3.

The evaluation experiments involved designated elderly subjects using long-term health care facilities (subjects 1-4) and patients using outpatient services (subjects 5-9). The purpose and methodology of the experiments was explained to the subjects. Thirteen subjects with sufficient understanding and willingness to participate (five males and eight females, $73.8 \pm 8.0$ years old, height $154.1 \pm 8.0 \mathrm{~cm}$, weight $58.2 \pm 10.4 \mathrm{~kg}$, and body mass index $24.6 \pm 3.0$ ) were selected for the study. Written informed consent was obtained from each subject. However, acceleration sensors were attached to only nine subjects, because the remaining subjects had physical difficulties. In addition to the motor function tests that evaluate subjects' rehabilitation progress at the long-term health care

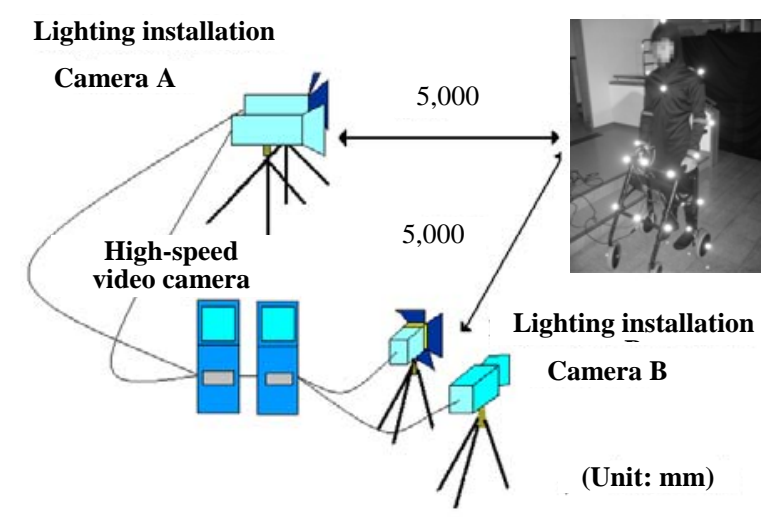

Fig. 3 Experimental apparatus. 
facility, we introduced a $10 \mathrm{~m}$ walk using the commercial walker. The motor function test comprised the TUG (timed "up and go") test, a 10 m walk (time and number of steps), standing from a wheelchair, time to stand on one foot with eyes open, the functional reach test, maximum step distance, and flexibility test [13]. The TUG test scores of patients with hip fracture are related to the type of walking aid they use [14]. The grip height was fixed at $80 \mathrm{~cm}$. The present study focused on the normal $10 \mathrm{~m}$ walk with and without the walker.

\section{Results}

First, normal walking with a commercial walker was assessed for the subjects in the control group. Stick diagrams (animations) of the subjects' normal walking motion were created from the images filmed by both high-speed video cameras and associated with the acceleration waveform simultaneously acquired from the acceleration sensors. Subjects moved from a static position after $2 \mathrm{~s}$ (Fig. 4) [13].

Large waveform changes were induced at points A, $\mathrm{C}$ and $\mathrm{D}$ as the right leg was moved vertically upward (along the $Z$-axis) and progressed in the horizontal direction (along the $X$-axis). The motion of the right leg increased along the $Z$-axis while decreasing along the $X$-axis direction. In video images, this motion occurred when the right foot landed. The left foot landed at the time indicated by point B. The waveforms induced by these motions appeared in a fixed cycle. The time elapsed between two landings of the right foot was $1.1 \mathrm{~s}$; within one cycle, the right foot landing was followed 0.5-0.6 s later by the left foot landing. The same cycle was observed in the lumbar acceleration. Although these magnitudes were smaller than in the leg acceleration, they were maximized when either foot landed.

The step width was determined from the speed of the body parts parsed from the high-speed video camera and the acceleration waveform. Regarding one step as the distance between the two successive points

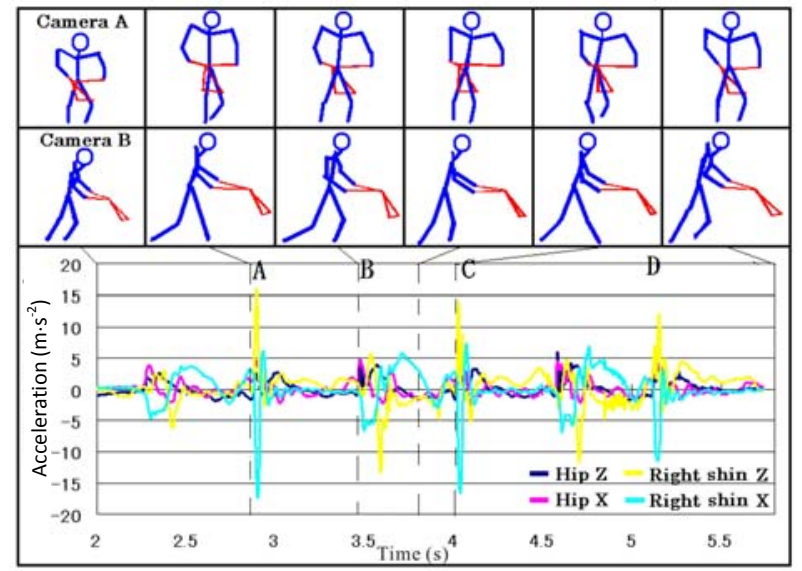

Fig. 4 Results of walking motion with commercial walker.

of ground contact of the right foot, the average step width was $86 \mathrm{~cm}$. By matching the video with the acceleration signal acquired from the tri-axial acceleration sensors, we found that general walking motion could be identified to some extent from the characteristics and size of the acceleration waveform.

\subsection{Rehabilitation Results}

At medical and nursing facilities, walking motion is visually evaluated by physical or occupational therapists, and therefore varies according to the observer's experience level and objectivity. In this section, we examine the use of acceleration measurements in analyzing the walking motions of elderly subjects before and after rehabilitation. We also assess the potential for introducing acceleration sensors to medical care and nursing facilities. Fig. 5 shows the acceleration waveform during vertical upswing and in the lateral direction (perpendicular to the direction of motion) in an elderly subject before rehabilitation. Prior to rehabilitation, the subject took two forward steps in a 1.3 s period. Walking motion data also revealed a peak vertical acceleration of 9.2 $\mathrm{m} \cdot \mathrm{s}^{-2}$, a lateral peak acceleration of $-6.8 \mathrm{~m} \cdot \mathrm{s}^{-2}$ and an irregular waveform (motion). The subject was then rehabilitated by muscle strength improvement training until no further care was required. The acceleration waveforms in the vertical and lateral directions after rehabilitation are presented in Fig. 6. The subject 


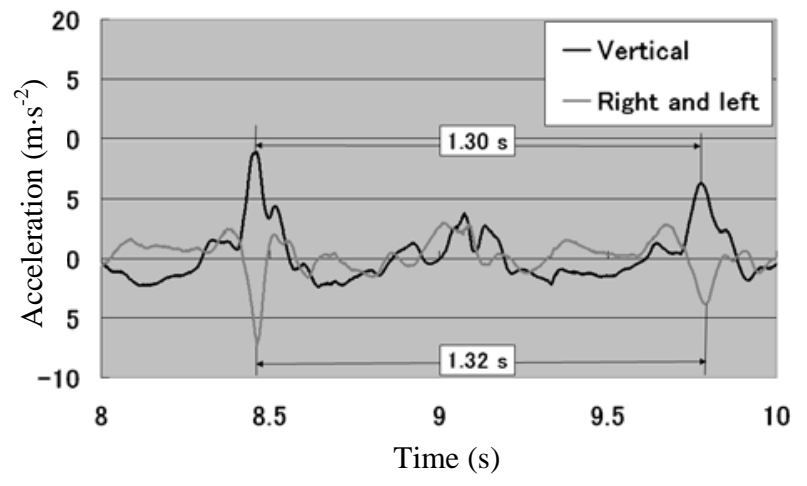

Fig. 5 Acceleration time histories for walking before rehabilitation [13].

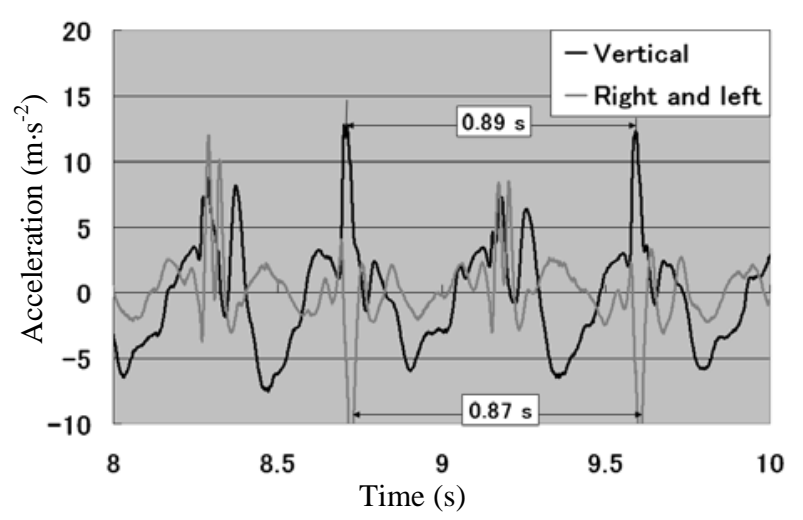

Fig. 6 Acceleration time histories for walking after rehabilitation [13].

now accomplished two forward steps in a 0.9 s period, and the peak vertical and lateral accelerations were improved to $13.3 \mathrm{~m} \cdot \mathrm{s}^{-2}$ and $12.2 \mathrm{~m} \cdot \mathrm{s}^{-2}$, respectively. These results demonstrate clear increases in walking speed and vertical and lateral acceleration following rehabilitation [13].

The average peak amplitudes in the acceleration waveforms of vertical and lateral movements performed by subjects 1-6 before and after rehabilitation are shown in Figs. 7 and 8, respectively. Apart from subject 6, the average accelerations were clearly increased after rehabilitation. Usually, walking is visually assessed by healthcare facility staff, using qualitative and relative evaluation standards. By the comparatively simple application of acceleration sensors to the lumbar region, we could base the results of rehabilitation on quantitative and absolute criteria.

Besides the magnitudes of the acceleration waveforms, all of the traditional test indicators, such

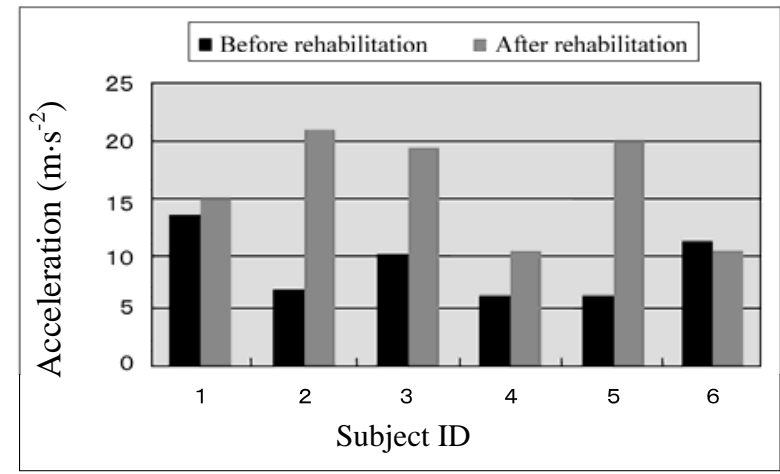

Fig. 7 Vertical accelerations before and after rehabilitation.

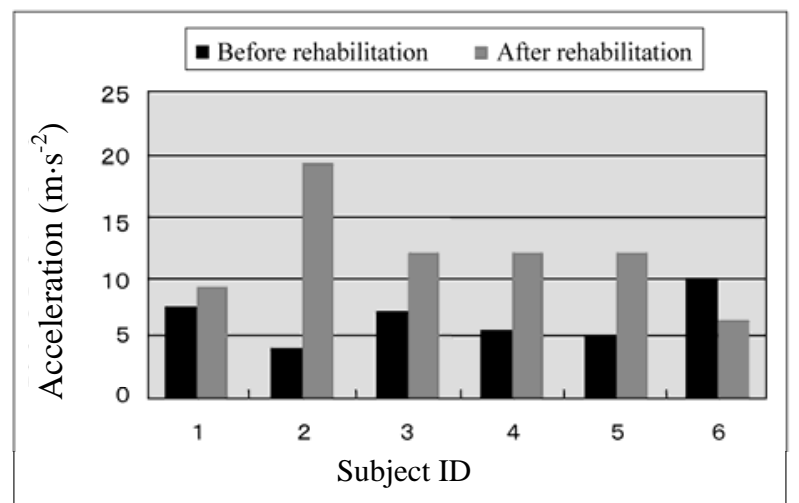

Fig. 8 Right and left accelerations before and after rehabilitation.

as walking speed, step length and time of standing on one foot with eyes open, were largely increased after rehabilitation. For the rehabilitated elderly subjects shown in Figs. 5 and 6, walking speed improved from $57.2 \mathrm{~m} \cdot \mathrm{min}^{-1}$ to $65.8 \mathrm{~m} \cdot \mathrm{min}^{-1}$, maximum step length increased from $55.6 \mathrm{~cm}$ to $62.5 \mathrm{~cm}$, and time of standing on one foot with eyes open dramatically improved from $10.3 \mathrm{~s}$ to $68.5 \mathrm{~s}$.

\subsection{Commercial Walker}

Fig. 9 shows the acceleration waveform of an elderly subject during normal walking without a commercial walker before rehabilitation. The acceleration waveform generated by the same subject using a commercial walker is presented in Fig. 10. In healthy subjects, the lumbar acceleration began to increase when the foot first contacted the ground (see the range of normal walking motions in Fig. 4). The step width was comparatively evenly timed at $0.55 \pm 0.02 \mathrm{~s}$, and the 


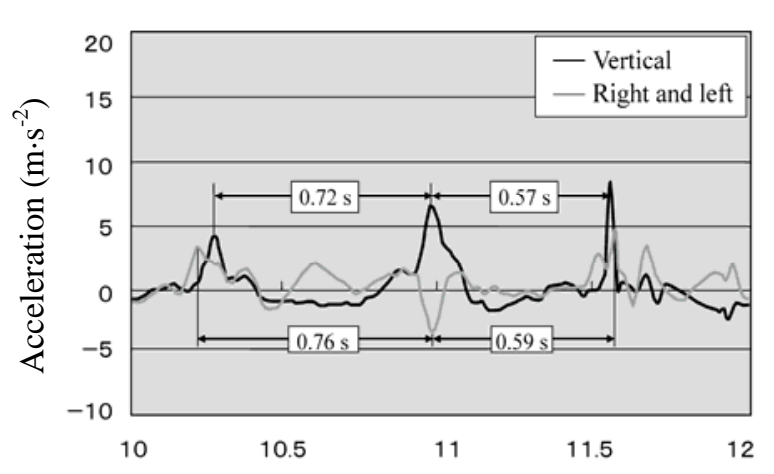

Time (s)

Fig. 9 Accelerations during unaided walking before rehabilitation [13].

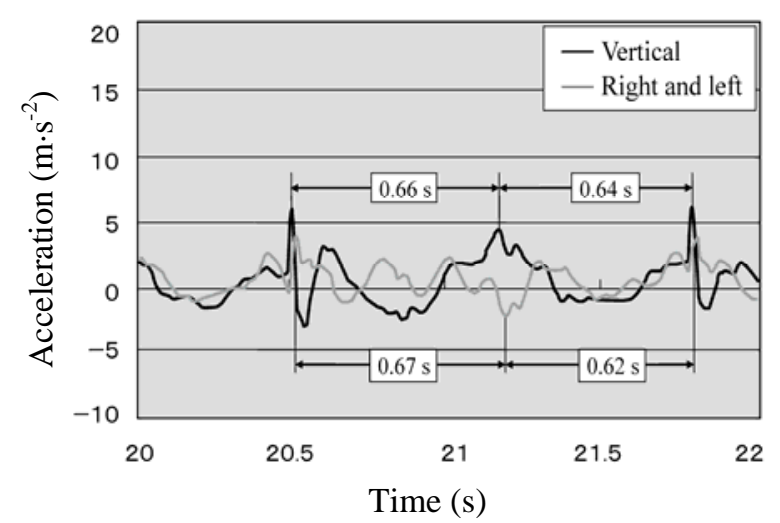

Fig. 10 Accelerations with a commercial walker before rehabilitation [13].

waveform was constant even when the lumbar region accelerated in the motion and vertical directions [13].

Comparing the acceleration waveforms in Fig. 9 with those of healthy subjects (Fig. 4), we observe that the accelerations of the elderly subjects were irregular in the vertical and lateral directions, and the step width timing was also non uniform. Using a commercial walker, the elderly subject achieved more consistent changes in the vertical and lateral accelerations, more regular step width timing, and improved walking motion (Fig. 10).

The acceleration waveforms generated in the vertical and lateral directions by this subject are presented in Figs. 11 and 12, respectively. Results are shown for (1) normal walking motions without a commercial walker and (2) walking motion with a commercial walker after rehabilitation. Comparing the

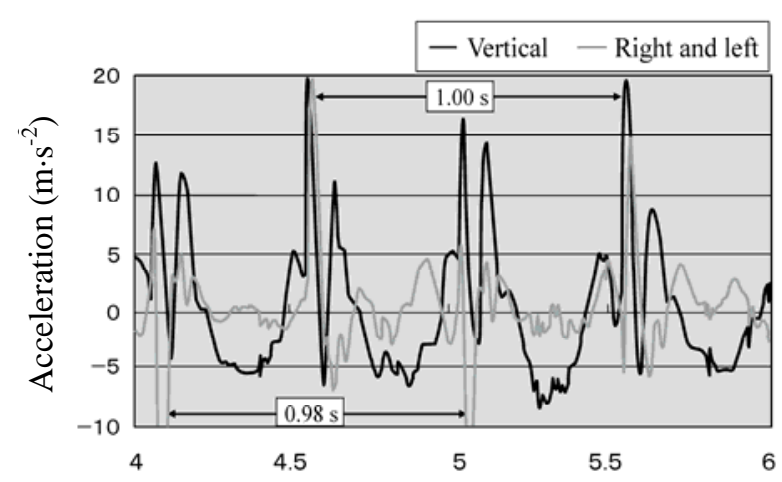

Time (s)

Fig. 11 Accelerations without a commercial walker after rehabilitation [13].

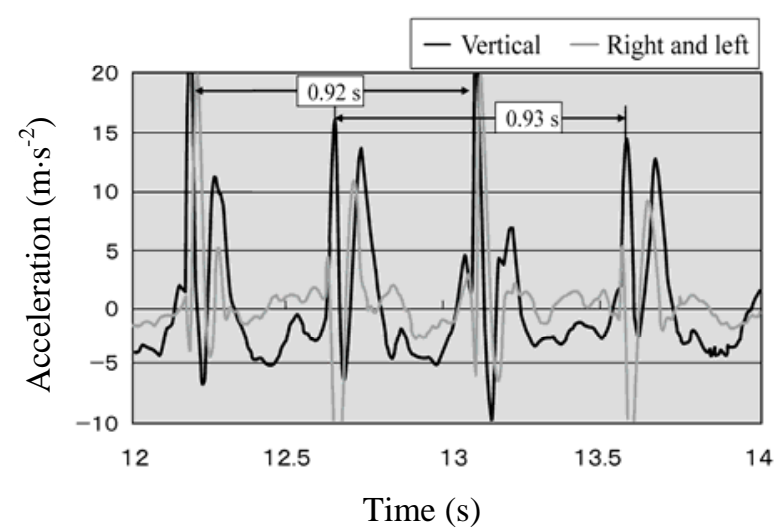

Fig. 12 Accelerations with a commercial walker after rehabilitation [13].

results in Figs. 11 and 12 to those in Figs. 9 and 10, we see that walking speed and acceleration was enhanced by rehabilitation, regardless of whether a commercial walker was used.

\subsection{Wheeled Walking Frame}

In this section, the system described in Section 2.1 is applied to the wheeled walking frame. Fig. 13 plots the acceleration waveforms in the vertical and lateral directions for an elderly subject without a wheeled walking frame. Fig. 14 plots the acceleration waveforms using a wheeled walking frame. Comparing the result of Fig. 14 to that of Fig. 13, we find that the vertical and lateral accelerations changed irregularly and the step width timing had become non uniform. However, the acceleration magnitudes decreased during the use of a wheeled walking frame, probably 


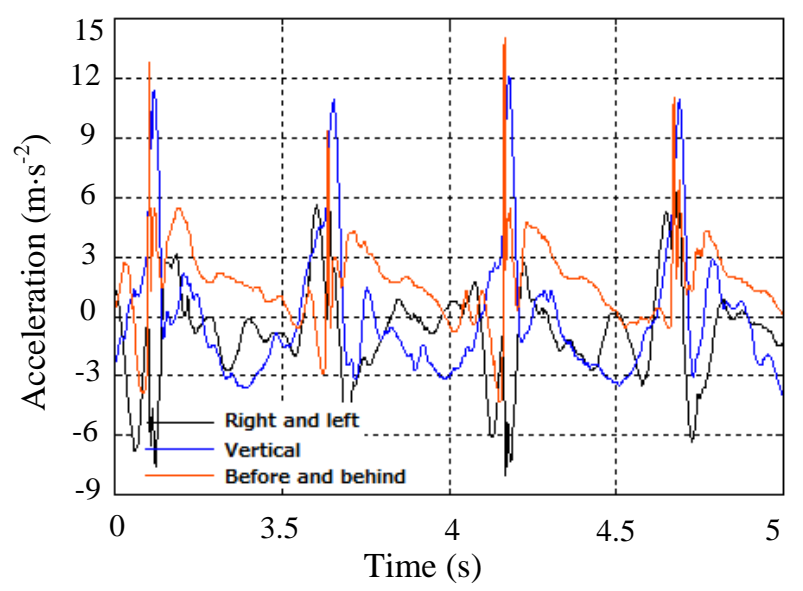

Fig. 13 Accelerations during unaided walking.

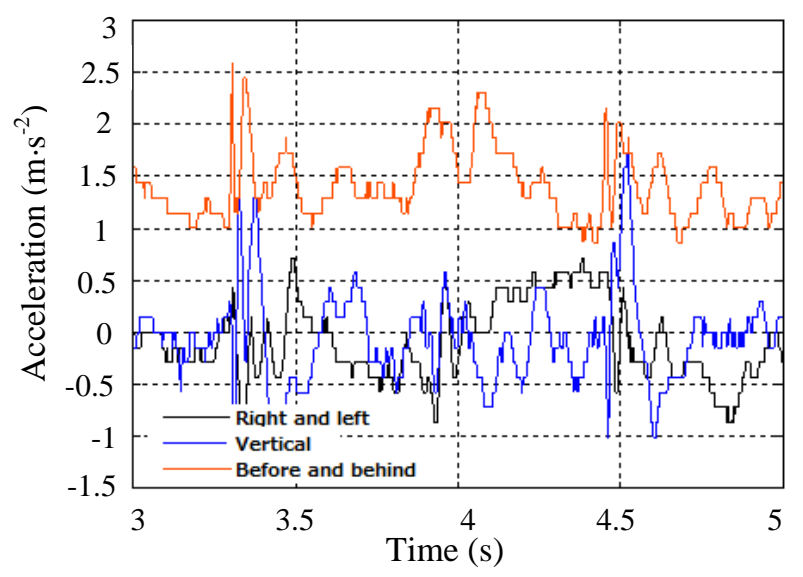

Fig. 14 Accelerations with a wheeled walking frame.

because the walker delivers a powerful lower walking propulsive force [13], which is enhanced by the wheels. The abovementioned trend was also observed in other subjects.

The acceleration spectrum of normal walking motion by each subject during unaided walking was recorded. The FFT (fast Fourier transformation) analysis reveals a sharp peak in the acceleration spectrum (Fig. 15). This feature characterizes walking motion and is consistent with the reports of other experimenters investigating walking motion [13]. Table 1 lists the averaged maximum peak acceleration frequencies during unaided walking, walking with a commercial walker, and walking with a wheeled walking frame. The peak acceleration frequency was lower when using the wheeled walking frame than when walking unaided or with a commercial walker.

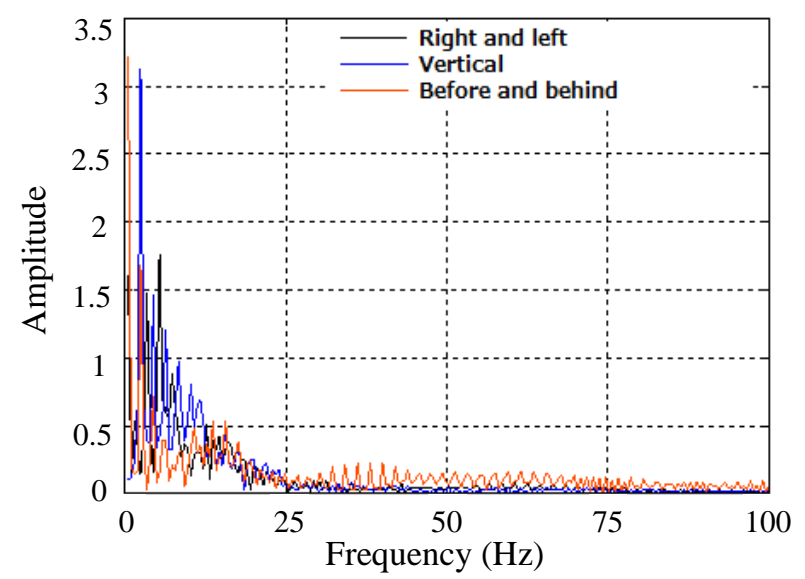

Fig. 15 FFT of accelerations during unaided walking.

Table 1 Averaged peak frequency.

\begin{tabular}{lccc}
\hline Averaged frequency (Hz) & $X-$ & $Y-$ & $Z-$ \\
\hline Accelerations during unaided walking & 5.4 & 8.2 & 2.7 \\
Accelerations with commercial walker & 2.2 & 5.8 & 2.1 \\
Accelerations with wheeled walking & 1.4 & 1.0 & 1.8 \\
frame & & & \\
\hline
\end{tabular}

\section{Discussion}

Research has revealed the comfortable and maximum walking speeds of adults aged 20-79 year [15]. The improvements in walking speed presented in Section 3.1 are attributable to increase propulsive force following rehabilitation. In contrast to walker use, rehabilitation also increased the step length, as reflected in rhythmic lateral and vertical movements. The same trend was observed in other subjects. Future micro-devices will be attachable to the body for extended periods, allowing patients to monitor their changes over time and thereby reach their self-training goals and improve their quality of life.

Four-wheeled walkers and the body postures of elderly users have been theoretically optimized for comfortable and steady walking [16]. Other studies have focused on partial weight-bearing gait while using conventional assistive devices [17], the stability and maneuverability of wheeled walking frames [18], and the impedance of lateral compensatory stepping movements by canes and walkers [19]. Therefore, we conclude that walkers absorb the vertical and lateral movements of the entire body during walking, while 
the inertial force of the walker wheels regulate the step width. When walking with a frame, the required energy is reduced by the absorption of the body's weight by the upper arms and reduction in the walking propulsive force. One study assessed the use of rolling walkers by older adults in senior living communities [20], while another study compared the gait characteristics between older rolling walker users and older potential walker users [21]. The propulsive force is reduced by the inertial force generated by the moving walker, which reduces the load on the legs and improves lateral balance. The result is a stable gait with constant acceleration and an even tempo.

To determine the effectiveness of the wheeled walking frame, we calculated the average RMS (root mean square) values of the peak acceleration amplitudes in the direction of motion ( $X$-axis, $Y$-axis and $Z$-axis), generated during foot-ground contact throughout the $10 \mathrm{~m}$ walk. The average RMS values of three successive steps undertaken by subjects 1-5 are shown in Fig. 16.

During normal walking, the posture of each foot is similar in every gait cycle. Foot acceleration is also relatively consistent. During the experiment, the three tri-axial acceleration sensor signals emitted from the lumbar region in the swing phase depended on the subject's walking speed. The gait cycles of normal walkers established a persistent relationship between the $X, Y$ and $Z$ sensor signals.

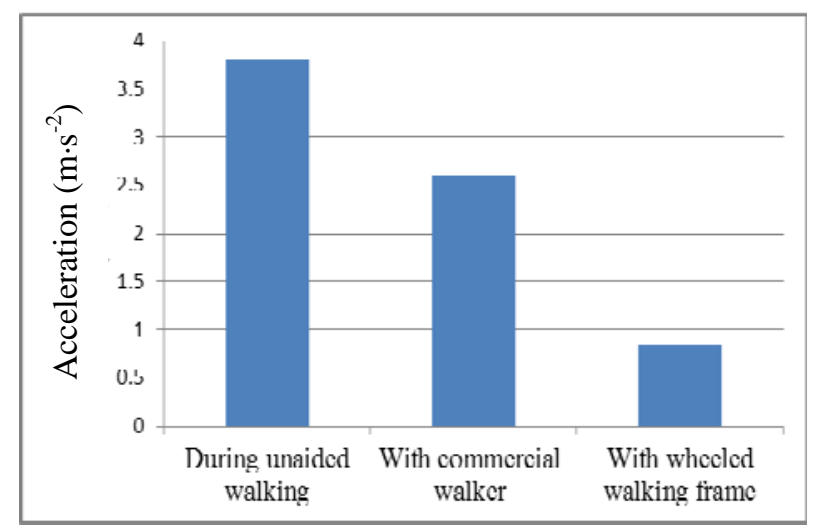

Fig. 16 RMS values of accelerations during unaided walking, with a commercial walker and with a wheeled walking frame.
RMS values of accelerations in $X$ - (ahead and behind), $Y$ - (right and left) and $Z$ - (vertical) directions during unaided walking are shown in Fig. 17 and those with a wheeled walking frame are shown in Fig. 18. Both figures show the smaller step widths and lower acceleration magnitudes by the inertial force of the wheels than those during unaided walking in $X$ direction. In addition, we found large acceleration magnitudes in designated subjects who could walk comparatively freely. These magnitudes decreased in subjects using a wheeled walking frame (Fig. 16). We also consider that when using a wheeled walking frame, the vertical and lateral movement of the entire

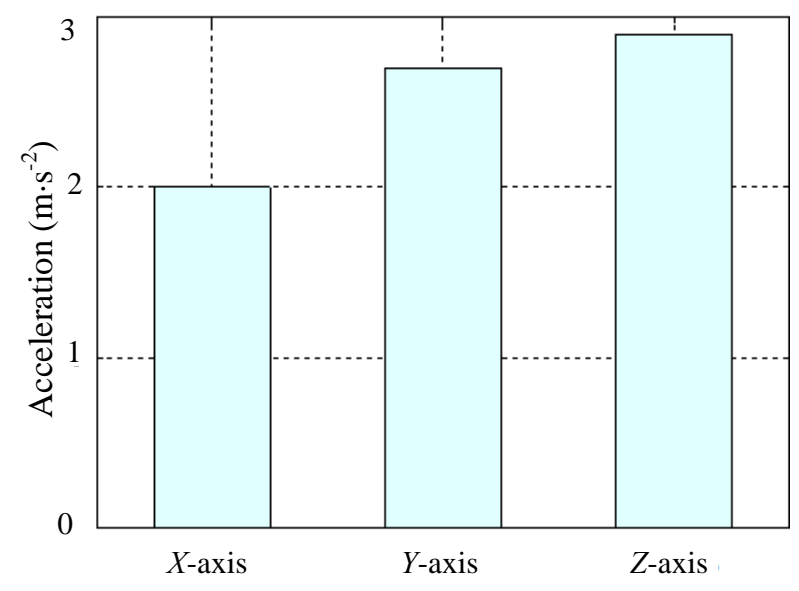

Fig. 17 RMS values of accelerations in $X$ - (ahead and behind), $Y$ - (right and left) and $Z$ - (vertical) directions during unaided walking.

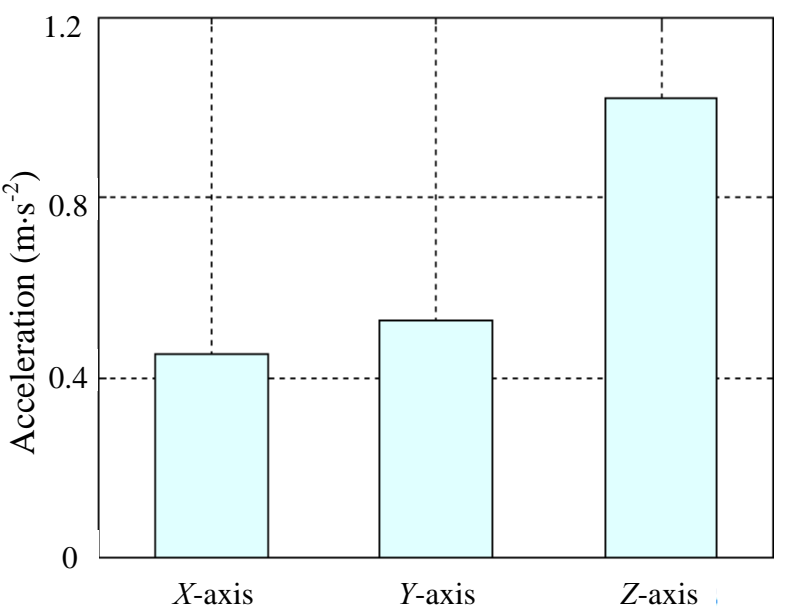

Fig. 18 RMS values of accelerations in $X$ - (ahead and behind), $Y$ - (right and left) and $Z$ - (vertical) directions with a wheeled walking frame. 
body during the walking motion is absorbed by the suspension of the walker, and that step width is regulated and reduced by the inertial force of the wheels.

\section{Conclusions}

Our preliminary experiment, which analyzed unstable movements during commercial walker use, employed two high-speed video cameras and three tri-axial acceleration sensors. The video images were correlated with the acceleration waveforms over a range of motions. The results indicate that motions (such as walking) can be determined to some extent by analyzing the acceleration variability and emerging peak values. We also evaluated the effectiveness of commercial walker use by elderly subjects taking a 10 $\mathrm{m}$ walk, TUG, standing, sitting and other activities. The effectiveness of using a commercial walker was quantified from the step width interval and the lateral and vertical acceleration magnitudes. This information was collected by sensors attached to the lumbar region.

Acceleration measurements were used to analyze walking motions before and after rehabilitation. We also explored the potential introduction of acceleration meters to medical and nursing facilities. Consequently, we found that rehabilitation efficacy could be based on quantitative and absolute criteria, in contrast to the subjective visual approaches currently adopted.

Furthermore, we used acceleration measurements to analyze the walking motions of subjects using a newly developed wheeled walking frame. The aim was to examine the effectiveness of the developed frame. Again, we found that by using the accelerometers, walking motion could be analyzed by quantitative and absolute criteria. Future study will relate traditional testing items to the results from the introduced acceleration sensors and gyro sensors.

\section{Acknowledgments}

We would like to thank President Mr. Yukio Kishi at Kishi Engineering Co., Ltd. for his cooperation during this research.

\section{References}

[1] Cook, A. H., and Hussey, S. H. 2002. Assistive Technologies: Principles and Practice. 2nd ed. St. Louis: Mosby.

[2] Bateni, H., and Maki, B. E. 2005. "Review Article: Assistive Devices for Balance and Mobility: Benefits, Demands, and Adverse Consequences.” Arch. Phys. Med. Rehabil. 86: 134-45.

[3] Cooper, R. A. 1995. Rehabilitation Engineering Applied to Mobility and Manipulation. New York: Taylor \& Francis.

[4] Hall, J., Clarke, A., and Harrison, R. 1990. "Guide Lines for Prescription of Walking Frames.” Physiotherapy 76 (2): 118-20.

[5] Crosbie, J. 1994. "Comparative Kinematics of Two Walking Frame Gaits.” J. Orthop. Sports Phys. Ther. 20 (4): 186-92.

[6] Alkjaer, T., Larsen, P. K., Pedersen, G., Nielsen, L. H., and Simonsen, E. B. 2006. "Biomechanical Analysis of Rollator Walking.” Biomed. Eng. Online 5 (2): 1-7.

[7] Tagawa, Y., Shiba, N., Matsuo, S., and Yamashita, T. 2000. "Analysis of Human Abnormal Walking Using a Multi-body Model: Joint Models for Abnormal Walking and Walking Aids to Reduce Compensatory Action.” J. Biomech. 33: 1405-14.

[8] Wright, D. L., and Kemp, T. L. 1992. "The Dual-Task Methodology and Assessing the Attentional Demands of Ambulation with Walking Devices.” Physical Therapy 72 (4): 306-12.

[9] Maki, B. E., Mcllroy, W. E., and Fernie, G. R. 2003. "Change-in-Support Reactions for Balance Recovery: Control Mechanisms, Age-Related Changes, and Implications for Fall Prevention.” IEEE Engineering in Medicine and Biology Magazine 22 (2): 20-6.

[10] Kim, S. C., Lee, M. H., Lee, S. Y., and Lee, Y. I. 2011. "Effect of Rollator Handle Type on Plantar Foot Pressure during Gait by Elderly Women.” J. Phys. Ther. Sci. 23: 729-31.

[11] Takenoshita, K., Nishiyama, T., Kawagoe, M., and Makiwawa, M. 2005. "Development of a Portable Acceleration Monitor Device and Its Clinical Application for the Quantitative Gait Assessment of the Elderly.” Presented at the 27th Annual International Conference of the Engineering in Medicine and Biology Society, Shanghai, China.

[12] Sekine, M., Akay, M., Tamura, T., Higashi, Y., and Fujimoto, T. 2004. "Fractal Dynamics of Body Motion in Patients with Parkinson's Disease.” J. Neural Eng. 1: 
8-15.

[13] Okamoto, S., Hirotomi, T., Aoki, K., and Hosomi, Y. 2012. Evaluation of Walking Motions with the Aid of Walkers Using Acceleration Sensors, Biomedical Engineering and Cognitive Neuroscience for Healthcare: Interdisciplinary Applications. Pennsylvania: IGI GLOBAL.

[14] Kristensen, M. T., Bandholm, T., Holm, B., Ekdahi, C., and Kehlet, H. 2009. "Timed Up \& Go Test Score in Patients with Hip Fracture Is Related to the Type of Walking Aid.” Arch. Phys. Med. Rehabil. 90: 1760-5.

[15] Bohannon, R. W. 1997. "Comfortable and Maximum Walking Speed of Adults Aged 20-79 Years: Reference Values and Determinants.” Age Ageing 26: 15-9.

[16] Takanokura, M. 2008. "Theoretical Optimization of Usage of Four-Wheeled Walker and Body Posture of Elderly Users for Comfortable Steady Walking." Presented at the 30th Annual International IEEE EMBS Conference, Vancouver, British Columbia, Canada.
[17] Youdas, J. W., Kotajarvi, B. J., Padgett, D. J., and Kaufman, K. R. 2005. "Partial Weight-Bearing Gait Using Conventional Assistive Devices.” Arch. Phys. Med. Rehabil. 86: 394-8.

[18] Anslow, R., Pinnington, L. L., Pratt, D. J., Spicer, J., Ward, C. D., and Weyman, N. 2001. "Stability and Manoeuvrability of Wheeled Walking Frames." Physiotherapy 87 (8): 402-12.

[19] Bateni, H., Heunga, E., Zettel, J., Mcllroy, W. E., and Maki, B. E. 2004. "Can Use of Walkers or Canes Impede Lateral Compensatory Stepping Movements?." Gait and Posture 20: 74-83.

[20] Liu, H. 2009. "Assessment of Rolling Walkers Used by Older Adults in Senior-Living Communities.” Geriatr. Gerontol. Int. 9 (2): 124-30.

[21] Liu, H., McGee, M., Wang, W., and Persson, M. 2009. "Comparison of Gait Characteristics between Older Rolling Walker Users and Older Potential Walker Users.” Archives of Gerontology and Geriatrics 48: 276-80. 